\title{
Preparação e caracterização eletroquímica de um eletrodo modificado com hexacianoferrato de manganês
}

\author{
E. Buffon (IC) ${ }^{1 *}$, M.L.S. Vasconcellos $(I C)^{1}$, D. Profeti $(P Q)^{1}$, L.P.R. Profeti (PQ)
}

1 Departamento de Química e Física, Universidade Federal do Espírito Santo, Alegre, ES, Brasil.

*e-mail: e_buffon@hotmail.com.br

\section{Resumo}

As análises eletroquímicas são amplamente utilizadas no controle de diversos processos industriais. Os eletrodos convencionais utilizados em tais análises possuem uma série de limitações, tais como a falta de reprodutibilidade, baixa sensibilidade, efeito de envenenamento da superfície do eletrodo e a cinética lenta de transferência de elétrons envolvidos na reação. Para contornar estas limitações a combinação entre o íon hexacianoferrato e os metais de transição mostra-se uma opção interessante para a preparação de eletrodos quimicamente modificados (EQMs), uma vez que, uma grande variedade desses compostos pode ser facilmente preparada a partir de substâncias químicas de custo reduzido, além do produto dessa combinação ter uma considerável eletrodeposição em diferentes superfícies. Neste contexto, este trabalho consistiu em sintetizar um filme de hexacianoferrato de manganês (MnHCF) sobre a superfície de um eletrodo de carbono vítreo (CV) e estudar o comportamento eletroquímico desse EQM em diferentes condições. Os resultados obtidos demonstraram que o processo de transferência de carga ocorre somente pelas reações eletroquímicas ocorridas na superfície do eletrodo e que o comportamento eletroquímico do EQM com MnHCF é influenciado pela força iônica da solução de eletrólito suporte, assim como a natureza dos diferentes cátions e ânions presentes na composição de tal solução.

\begin{abstract}
The electrochemical analyses are largely used at the control of a several industrial processes. Nevertheless, the traditional electrodes used on these analyses have some limitations, such as the low reproducibility, poor sensitivity, surface poisoning effect, and the slow kinetic of the electron transfer. In order to overcome these limitations, the modification of the electrode surface is a promising alternative. The combination between hexacyanoferrate and transition metals is an interesting option for preparing chemically modified electrodes (CMEs), since a large variety of these compounds can be easily synthetized by using chemical substances with low cost, as well as the product of this reaction has a considerable electrodeposition at different surfaces. Thus, in this work was prepared a film of manganese hexacyanoferrate (MnHCF) on a glassy carbon electrode, and its electrochemical behavior in different conditions was investigated. The results showed that the charge transfer process are controlled just by the electrochemical reactions on the electrode surface, and the electrochemical behavior of the MnHCF is influenced by the ionic force of the electrolyte solution, as well as by the nature of the different cations and anions present in the solution.
\end{abstract}

Keywords (Palavras chaves): Eletrodo Quimicamente Modificado, Hexacianoferrato de Manganês. 


\section{Introdução}

Os Eletrodos Quimicamente Modificados (EQM's) têm sido amplamente utilizados em diversas metodologias analíticas. O termo EQM's foi primeiramente adotado por Murray em 1975, que verificou que este permitia o estudo de espécies quimicamente ativas, deliberadamente imobilizadas em superfícies de eletrodos convencionais $\mathrm{Au}, \mathrm{Pt}$, filme de $\mathrm{Hg}$, carbono vítreo, entre outros) [1]. Além disso, Murray verificou que limitações em eletrodos convencionais, tais como (i) envenenamento da superfície por adsorção de espécies durante reações eletroquímicas e (ii) aplicação de elevados sobrepotenciais para garantir a oxidação de algumas espécies com baixa velocidade de reação, poderiam ser contornadas pela presença de substâncias imobilizadas na superfície eletródica [2].

A escolha do material para o eletrodo base é importante para o preparo do Eletrodo Quimicamente Modificado (EQM), uma vez que este deve apresentar características eletroquímicas adequadas para 0 método de imobilização desejado [3].

A modificação química da superfície dos eletrodos pode ser feita através da incorporação simples de substâncias adsorventes. Um exemplo de espécie modificadora de superfície de eletrodos são os hexacianoferratos de metais (MHCF) os quais são análogos do Azul da Prússia (AP). A formação de filmes finos de AP foi reportada pela primeira vez por Neff [4].

Os MHCF apresentam alta reversibilidade eletroquímica, e formam complexos com grande variedade de metais de transição. Como exemplo temos o Hexacianoferrato de Manganês (MnHCF), um análogo interessante do $\mathrm{AP}$, o qual ainda tem sido pouco estudado [5]. Neste contexto, o objetivo deste trabalho consistiu em sintetizar um filme de hexacianoferrato de manganês (MnHCF) sobre a superfície de um eletrodo de carbono vítreo (CV) e estudar o comportamento eletroquímico desse EQM em diferentes condições.

\section{Materiais e métodos}

O Hexacianoferrato de Manganês (MnHCF) foi sintetizado utilizando a técnica de voltametria cíclica com o auxílio de um potenciostato portátil PalmSens modelo EmStat 3+, operado pelo software PSTrace 4.4, juntamente com uma célula eletroquímica constituída por um eletrodo de trabalho de carbono vítreo (disco com $3 \mathrm{~mm}$ de diâmetro), um fio de platina como contra-eletrodo e um eletrodo de $\mathrm{Ag} / \mathrm{AgCl}$ como referência, o qual foi utilizado em todos experimentos deste trabalho.

Antes da formação do filme, o eletrodo de carbono vítreo (CV) foi polido com uma suspensão de alumina $0,05 \mu \mathrm{m}$ e lavado com água ultrapura para a remoção das partículas. Posteriormente, o eletrodo de CV foi imerso em uma solução de $\mathrm{K}_{3} \mathrm{Fe}(\mathrm{CN})_{6}$ e uma solução de $\mathrm{MnSO}_{4} \cdot \mathrm{H}_{2} \mathrm{O}$, numa razão molar de 1:1 (ambas preparadas numa solução de eletrólito suporte de $\mathrm{KCl}$ $\left.0,1 \mathrm{Mol} \mathrm{L}^{-1}\right)$. Para a eletrossíntese do filme foi utilizada a técnica de voltametria cíclica aplicando-se 40 ciclos sucessivos de varredura de potenciais, em um intervalo entre $-0,2$ a 1,2 V, a uma velocidade de varredura de $50 \mathrm{mV} \mathrm{s}^{-1}$.

Neste trabalho todos os reagentes utilizados foram de grau analítico, sendo eles: $\mathrm{KCl}, \mathrm{KNO}_{3}, \mathrm{NaCl}, \mathrm{NaNO}_{3}$, $\mathrm{K}_{2} \mathrm{SO}_{4}$ e LiCl. Todas as soluções aquosas utilizadas foram preparadas com água ultrapura de um sistema de purificação MEGAPURITY MEGA RO/UP.

\section{Resultados e discussões}

O filme de MnHCF foi formado por eletrodeposição sob superfície de eletrodo de carbono vítreo (CV), onde o eletrodo foi submetido a sucessivos ciclos entre os potenciais $-0,2 \mathrm{~V}$ a $1,2 \mathrm{~V}$ como mostrado na Figura 1. Nesta figura observa-se o aumento de duas correntes de pico catódicos e duas correntes de pico anódicos, localizadas num intervalo de potencial de $0,2 \mathrm{~V}$ a $1,0 \mathrm{~V}$, ao final de cada ciclo de varredura. 


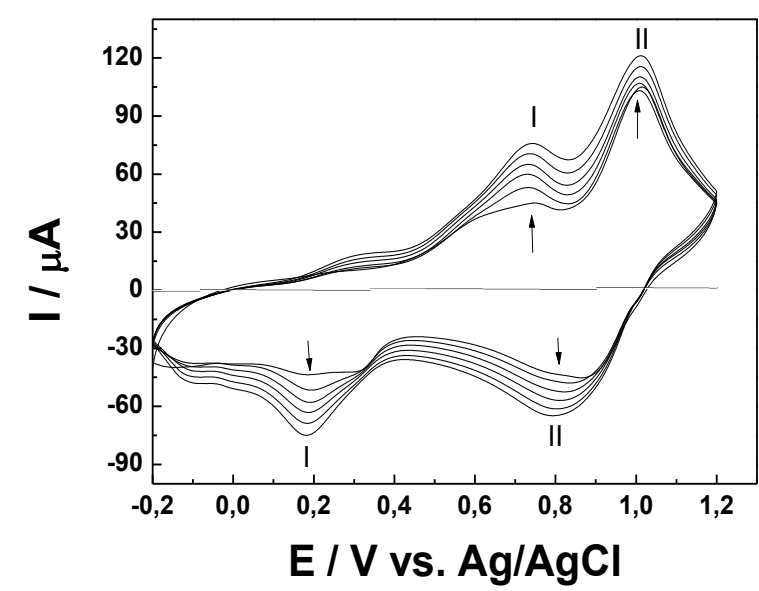

Figura 1: Crescimento do filme MnHCF em KCl 1,0 $\times 10^{-1} \mathrm{~mol} \mathrm{~L}^{-1}$ a $50 \mathrm{mV} \mathrm{s}^{-1}$

Ao analisar a Figura 1 observa-se que o crescimento do filme de MnHCF sobre a superficie do eletrodo de CV se manifesta na forma de dois picos anódicos e dois picos catódicos. De acordo com Eftekhari [6], a existência dos picos redox em I podem ser atribuídos ao sistema $\mathrm{Fe}[(\mathrm{CN}) 6]^{3-} / \mathrm{Fe}[(\mathrm{CN}) 6]^{4-}$. Outro aspecto observado nos voltamogramas cíclicos de formação do filme de MnHCF é a presença de um par de picos redox reversível (picos II) na faixa de potencial entre $0,8 \mathrm{~V}$ a $1,0 \mathrm{~V}$. Tais picos podem ser atribuídos a transição redox $\mathrm{Mn}^{2+} / \mathrm{Mn}^{3+}$ [5].

Após a formação do filme de MnHCF, verificou-se o comportamento eletroquímico do mesmo na presença de soluções $\mathrm{KCl}$ em diferentes concentrações, com o intuito de investigar como a força iônica da solução pode influenciar no comportamento eletroquímico do filme, conforme indicado na Figura 2.

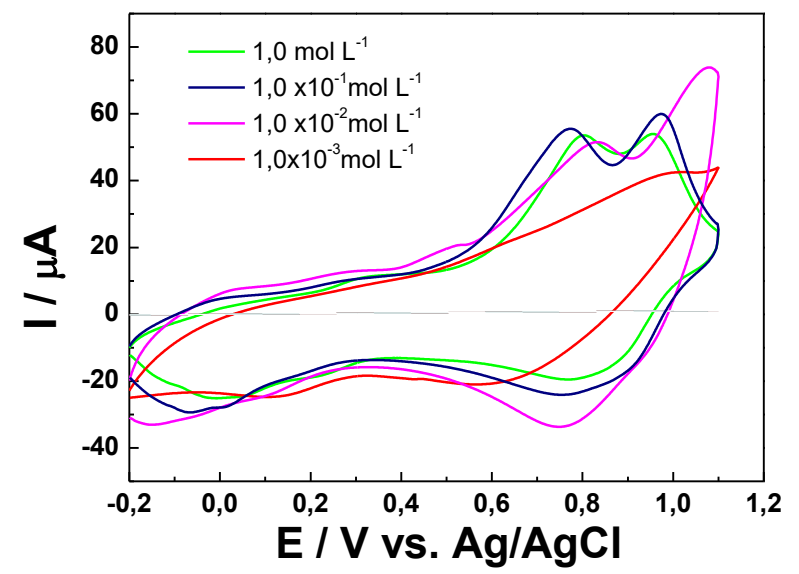

Figura 2: Comportamento ciclovoltamétrico do filme MnHCF na presença de $\mathrm{KCl}$ em diferentes concentrações.
Nesta figura, nota-se que o voltamograma cíclico do filme de MnHCF em soluções de $\mathrm{KCl}$ com menores concentrações do íon potássio, possui menor definição dos picos referentes aos processos redox bem como menor carga voltamétrica, o que pode ser atribuído às limitações associadas com a propagação de cargas no interior da estrutura do complexo, predominante em soluções com menores concentrações de $\mathrm{K}^{+}$[6].

Para manter a eletroneutralidade durante as reações eletroquímicas, os íons do eletrólito suporte se difundem para o interior do retículo cristalino do complexo, causando modificações no perfil voltamétrico do eletrodo modificado com MnHCF. Com a finalidade de verificar a influência da natureza de diferentes cátions e ânions no comportamento eletroquímico do filme de MnHCF, o mesmo foi investigado na presença de diferentes eletrólitos e os resultados estão mostrados na Figura 3.

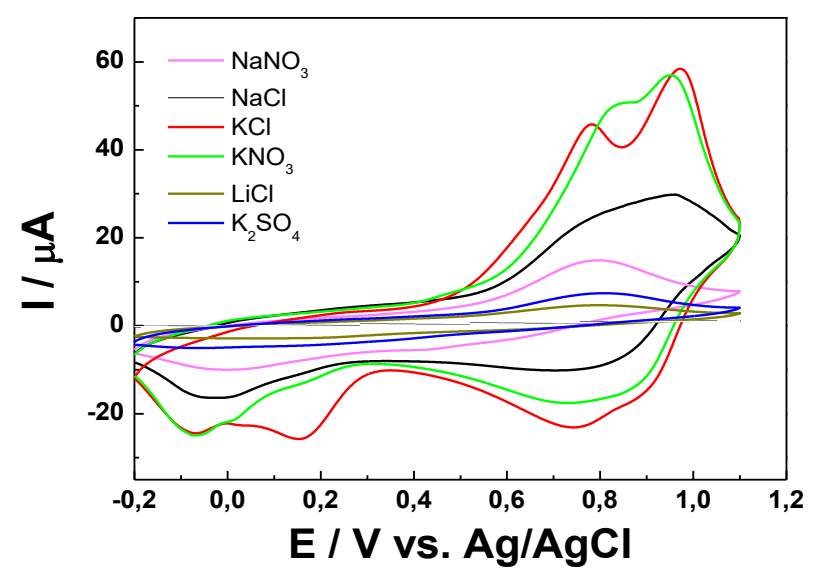

Figura 3: Comportamento ciclovoltamétrico do filme MnHCF na presença de diferentes eletrólitos suporte com velocidade de varredura de potenciais de $20 \mathrm{mV} \mathrm{s}^{-1}$.

Nesta figura, observa-se que o comportamento eletroquímico do complexo sofre algumas alterações na presença dos cátions $\mathrm{K}^{+}, \mathrm{Na}^{+}$, $\mathrm{Li}^{+}$, os quais apresentam raio iônico de 276, 204 e 148 pm respectivamente. De acordo Scholz e Dostal [7], os cátions com maior raio iônico penetram mais facilmente nos canais presentes na estrutura do complexo devido a menor esfera de hidratação, facilitando o processo de inserção/deinserção de espécies. Desta forma, o $\mathrm{K}^{+}$participa efetivamente dos processos superficiais de oxidação e redução do filme, como pode ser comprovado pela maior intensidade das correntes de pico nos eletrólitos 
$\mathrm{KCl}$ ou $\mathrm{KNO}_{3}$ em relação aos demais eletrólitos estudados, contendo $\mathrm{Na}^{+}$e $\mathrm{Li}^{+}$.

O perfil voltamétrico do filme de MnHCF na presença do eletrólito de $\mathrm{K}_{2} \mathrm{SO}_{4}$ apresentou os menores valores de corrente de pico e carga voltamétrica dentre os eletrólitos estudados. Esse comportamento se deve à possível interação do ânion $\mathrm{SO}_{4}{ }^{2-}$ com o filme, ocasionando a possível degradação do mesmo.

Com a finalidade de verificar como ocorre o controle dos processos eletroquímicos na superfície do EQM, submeteu-se o mesmo a diferentes velocidades de varredura em uma solução de eletrólito suporte de $\mathrm{KCl}$ $1,0 \times 10^{-1} \mathrm{~mol} \mathrm{~L}^{-1}$. As Figuras 4 e 5 indicam a relação entre as correntes de pico anódicas e catódicas tanto com a velocidade de varredura como com a raiz quadrada da velocidade de varredura, respectivamente.

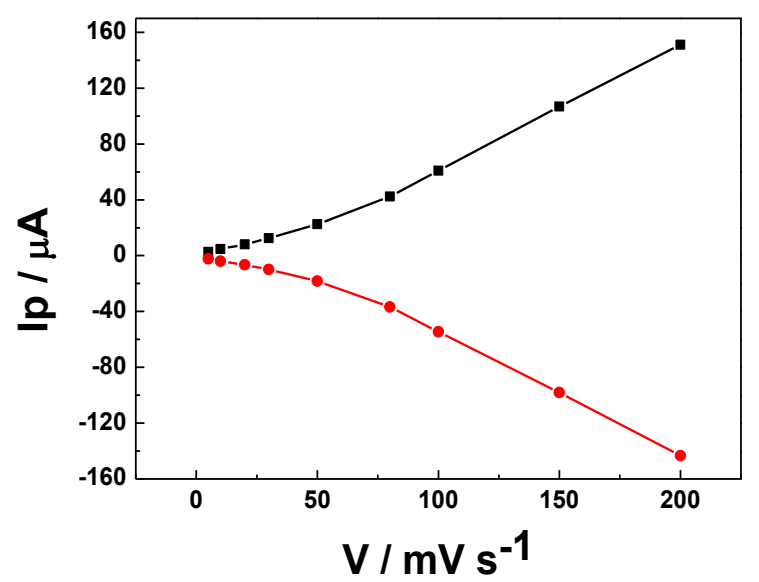

Figura 4: Relação das correntes de pico anódica e catódica com velocidade de varredura.

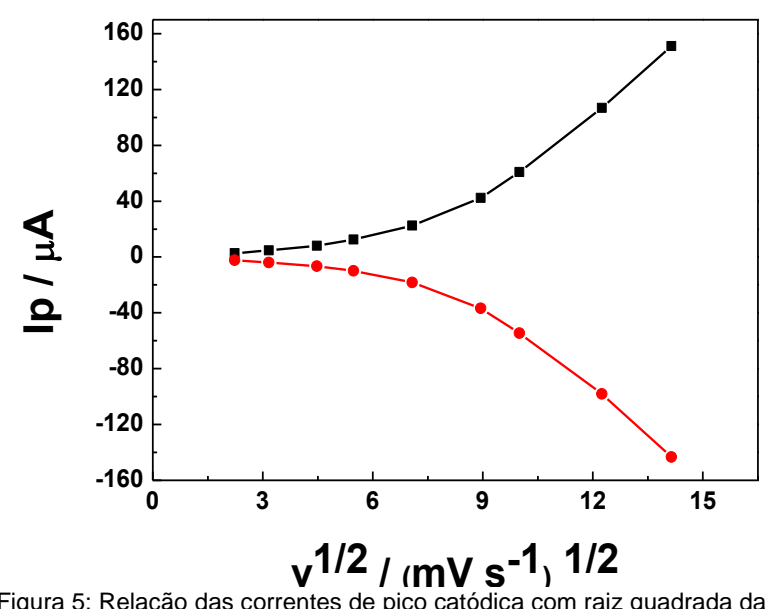

Figura 5: Relação das correntes de pico catódica com raiz quadrada da velocidade de varredura.
$\mathrm{Na}$ Figura 4 observa-se que as correntes de pico aumentam linearmente com o aumento da velocidade de varredura, indicando que a cinética do processo de transferência de carga é controlada somente pela reação eletroquímica na superfície do eletrodo e não é limitado pela difusão das espécies no interior do filme. Este comportamento pode ser confirmado através da Figura 5 , onde se observa que a relação entre a raiz quadrada da velocidade de varredura com o valor da corrente de pico não apresentou um comportamento linear, indicando que não há um controle difusional no mecanismo de transferência de carga [8].

\section{Conclusão}

O método empregado para a eletrossíntese do EQM foi adequado, uma vez que obteve-se uma considerável reprodutibilidade durante a eletrodeposição do complexo de MnHCF na superfície do eletrodo de CV.

Com os estudos realizados para a caracterização eletroquímica do EQM com MnHCF constatou-se que o processo de transferência de carga ocorre somente pelas reações eletroquímicas ocorridas na superfície do EQM.

Observou-se ainda que o comportamento eletroquímico do filme de MnHCF é influenciado pela força iônica da solução eletrolítica, assim como a presença de diferentes cátions e ânion na composição da mesma.

\section{Agradecimentos}

Os autores agradecem à Fundação de Amparo a Pesquisa do Espírito Santo (FAPES) pelo auxílio concedido e ao Programa Institucional de Iniciação Cientifica (PIIC) da Universidade Federal do Espirito Santo.

\section{Referências}

[1] - SOUZA, M. F. B. Quím. Nova, v.20, p.191-195, 1997.

[2] - QUINTINO, M. S. M. Desenvolvimento de sensores eletroquímicos associados a batch injection 
analysis (bia) para aplicações analíticas, 2003, 112f, Tese (Doutorado em Ciências - Química Analítica) Instituto de Química, Universidade de São Paulo, 2003.

[3] - PEREIRA, A. C.; SANTOS, A. S.; KUBOTA, L. T. Quím. Nova, v.25, p.1012-1021, 2002.

[4] - NOSSOL, E. Novos eletrodos construídos a partir de diferentes nanoestruturas de carbono, 2009, 129f, Dissertação (Mestrado em Química) Departamento de Química, Universidade Federal do Paraná, 2009.

[5] - LIU, Y.; YAN, Y.; SHEN, H. Chin. J. Chem., v.23, p.1165-1172, 2005.

[6] - EFTEKHARI, A. Talanta, v.55, p.395-402, 2001.

[7] - SCHOLZ, F.; DOSTAL, A. Angrew. Chem. Int. Ed. Engl., v.34, p.2685-2687, 1995.

[8] - ARDUINI, F.; CASSINI, A.; AMINE, A.; RICCI, F.; MOSCONE, D.; PALLESCHI, G. J. Electroanal. Chem., v.626, p.66-74, 2009. 